\title{
Design of Log Periodic Dipole Array Antenna Ranging from 30 to $150 \mathrm{MHz}$
}

\author{
Upendra Subedi $^{*}{ }^{,}$, Rabin Nepal $^{1}$, Rhimesh Lwagun ${ }^{1}$, and Sanjay Rijal ${ }^{1}$ \\ ${ }^{1}$ Department of Electronics and Computer Engineering, Thapathali Campus, Institute of Engineering, \\ Tribhuvan University, Kathmandu, Nepal \\ *Corresponding Email: tha073bex347@tcioe.edu.np
}

\begin{abstract}
This paper describes construction of 16 element frequency independent and high bandwidth Log Periodic Dipole Array antenna which works within the frequency range of 30 to $150 \mathrm{MHz}$. The antenna of gain $8 \mathrm{dBi}$ is designed by calculating the parameters from Matlab. Matlab program is used to calculate the parameters like length of elements, separation between elements, theoretical impedance, standing wave ratio, etc. Thus, the designed antenna was fabricated. The antenna element is a cylindrical aluminum pipe of diameter $9 \mathrm{~mm}$ and the boom used is rectangular antenna pipe of dimension 1"X1". Fabrication also includes other materials like M-seal, zip tie, etc for physical stability of the antenna. Log Periodic Dipole Array antenna design described in this paper is fed with $75 \mathrm{ohm}$ coaxial cable to 1:1 balun. Balun is also used for impedance matching between the antenna and coaxial cable. Antenna analyzer is used to measure Standing Wave Ratio and Impedance which are found to be 1.623 and $71.5625 \mathrm{ohm}$ respectively. The antenna can be a receiver or a transmitter type for operating with the signals within Very High Frequency band.
\end{abstract}

Keywords: Antenna, Frequency Independent, Log Periodic Dipole Array, Very High Frequency

\section{INTRODUCTION}

Antenna is a device that converts electric signal into electromagnetic signal and vice versa. Communication through unguided medium uses electromagnetic signals and antennas. Nature and size antenna vary for according to frequency of radio wave that is used for communication.

Among various types of antennas, LPDA antenna is used for transmitting signals of higher frequency range. LPDAs are frequency independent, broadband, directional antennas that consists of element varying logarithmically in both length and spacing between the elements. The length of dipoles are associated with half the wavelength of receiving signal. LPDA is used for Television, point to point communication, as receiving antenna in receiving station, Radio and many more. Depending on its design parameters, LPDA can be operated across a wide range of frequencies in VHF as well as UHF and over this range the gain, feed-point impedance, front-to-back ratio, etc. remains more or less constant. LPDA exhibits relatively low standing wave ratio (SWR) over a wide range of frequencies usually 2:1 and has frequency independent performance as well. [1] R.H. DuHamel first designed Log 
periodic antenna in 1957. [2] The first work on LPDA was published in 1960 by D.E. Isbell. However, the practical design was first published in 1961 by CARREL [3].

Designing of LPDA antenna includes calculation of parameters like number and size of antenna elements, boom spacing, and separation between antenna elements. All calculations are done using Matlab. Impedance and standing wave ratio (SWR) of antenna was measured with vector network analyzer also known as Antenna analyzer.

\section{MATHEMATICAL MODELING}

The elements of LPDA are excited with 180 degree phase shift with length and spacing between the elements varying in accordance with equation 1 and 2. [4] Here, length of $i^{\text {th }}$ element of antenna is $L_{i}$ and spacing between $i^{\text {th }}$ and $(i+1)^{\text {th }}$ element is denoted by $\mathrm{R}_{\mathrm{i}}$.

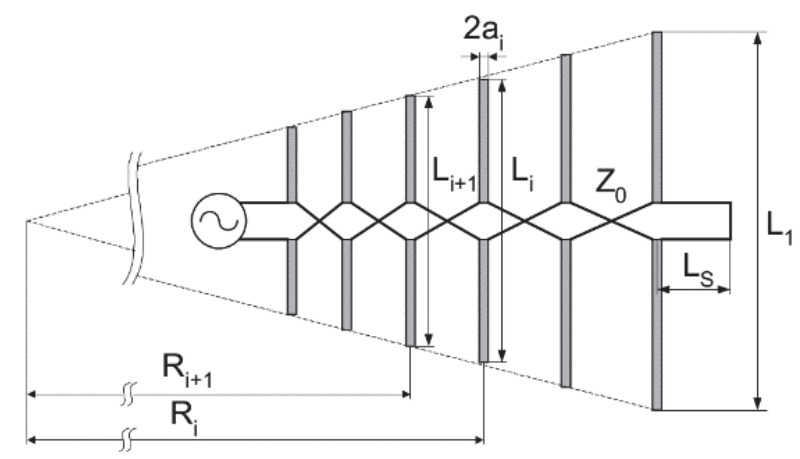

Figure 1: General structure of LPDA.

$$
\begin{gathered}
L_{i+1}=\tau L_{i} \\
R_{i+1}=\tau R_{i} \\
\sigma=\frac{R_{i}-R_{i-1}}{2 \cdot L_{i}}
\end{gathered}
$$

Where $\tau$ is design constant called periodicity $(0<\tau<1)$ and $\sigma$ is relative spacing of the elements.

The design input parameters are the nominal input resistance $\left(R_{0}\right)$, the desired gain $(G)$ relative to isotropic radiator, and frequency range expressed as the lower $\left(f_{1}\right)$ and upper $\left(f_{2}\right)$ operating frequencies.

In the design $\mathrm{R}_{0}$ is taken $50 \mathrm{ohm}$ to achieve VSWR less than 1.5. The lower cutoff frequency ( $\mathrm{f}_{1}$ ) is 30 $\mathrm{MHz}$ and upper cutoff frequency $\left(\mathrm{f}_{2}\right)$ is $150 \mathrm{MHz}$ with a gain of $8 \mathrm{dBi}$. The gain of antenna is constant and taken as reference for estimating values of periodicity $(\tau)$ and relative spacing $(\sigma)$. 


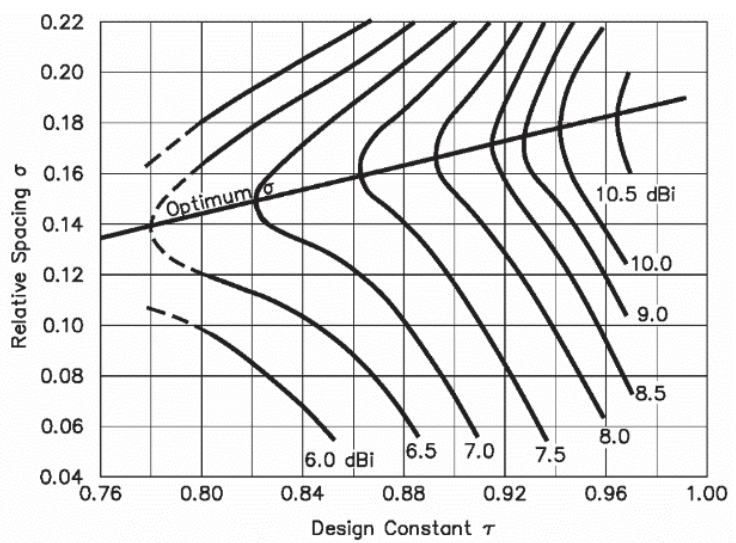

Figure 2: Relative spacing vs. periodicity graph. For the gain of $8 \mathrm{dbi}$, relation between sigma and tau is as equation (4)

The corresponding values $\tau$ and $\sigma$ for Gain $(\mathrm{G})=8 \mathrm{dBi}$ is determined from the relative spacing vs periodicity graph exhibiting linear relationship of $\tau$ and $\sigma$ as:

$$
\sigma=0.243 \tau-0.051
$$

Relative bandwidth (B) of the system was calculated as,

$$
B=\frac{f_{2}}{f_{1}}
$$

Active region bandwidth $\left(\mathrm{B}_{\mathrm{ar}}\right)$, structure bandwidth $\left(\mathrm{B}_{\mathrm{s}}\right)$ was calculated as,

$$
\begin{gathered}
B_{a r}=1.1+7.7(1-\tau)^{2} \frac{4 \sigma}{1-\tau} \\
B_{s}=B * B_{a r}=\frac{f_{2}}{f_{1}} * B_{a r}
\end{gathered}
$$

The required number of dipole elements $(\mathrm{N})$ and length of longest element which corresponds to the frequency of $30 \mathrm{MHz}$ were calculated as,

$$
\begin{gathered}
N=1+\left(\frac{\log \left(B_{s}\right)}{\log (1-\tau)}\right) \\
L_{1}=\frac{C}{2 f}
\end{gathered}
$$

Where $\mathrm{C}$ is the speed of EM waves in vacuum. The spacing between two elements is given by,

$$
R i-R i+1=(L i-L i+1) * \frac{4 \sigma}{1-\tau}
$$

For higher front-to-back ratio at the lowest frequency, the antenna feeder is shorted at a distance $\mathrm{L}_{\mathrm{s}}$ behind the longest element. The short acts as a reflector; its distance from the longest element is given by. [4]

$$
L s=\frac{\lambda}{4}
$$


The distance between booms is calculated as,

Where, $b$ is the diameter of feeder tube and

$$
d f=b \cosh \left(\frac{Z_{0}}{120}\right)
$$

$\mathrm{Z}_{0}$ is the feeder characteristic impedance ensuring the lowest possible VSWR [4].

$$
Z_{0}=\frac{Z_{0}^{2} \sqrt{\tau}}{8 Z_{a v} \sigma}+R_{0} \sqrt{\frac{\left(R_{0} \sqrt{\tau}\right)^{2}}{\left(8 Z_{a v} \sigma\right)^{2}}+1}
$$

Where,

$$
Z a v=120 \ln S-2.25
$$

The slimness factor can be calculated as

$$
S=\frac{L_{i}}{2 a_{i}}
$$

Where $2 a_{i}$ is the diameter of $i^{\text {th }}$ element and $L_{i}$ is its length.

\subsection{CALCULATION OF SWR}

When antenna receives signals transmitted from transmitter, some portion of the signal gets reflected back and the coefficient of reflection $(\rho)$ is calculated as,

$$
\rho=\left|\frac{Z_{1}-Z_{2}}{Z_{1}+Z_{2}}\right|
$$

Where, $\rho=$ reflection coefficient and $Z_{1}$ and $Z_{2}$ are impedances of mismatched lines. Using the value of reflection coefficient, Standing Wave Ratio (SWR) is,

$$
S W R=\frac{1+\rho}{1-\rho}
$$

Ideally, SWR is 1:1 which means that there is no power being reflected back to the source. Practically, a SWR of 1.2:1 is considered excellent in most cases. [5] At SWR of 2:1, approximately 10\% of the power is reflected back to the source. Not only does a high VSWR mean that power is being wasted, the reflected power can cause problems such as heating cables or causing amplifiers to fold-back.

Calculation of Return Loss and Mismatch Loss:

$$
\begin{array}{r}
\text { Return Loss }=-20 \log \left[\frac{V S W R-1}{V S W R+1}\right] \\
\therefore \text { Return Loss }=-20 \log \rho \\
\text { Mismatch loss }=-10 \log \left(1-\rho^{2}\right)
\end{array}
$$

Receiving log-periodic dipole array antenna was designed using the above mathematical calculations. 


\section{PARAMETER CALCULATION}

The values of $\tau$ and $\sigma$ taken from graph are used to calculate length and spacing of elements.

The parameter calculation for designing antenna is done on Matlab. Value of $\tau, \sigma$, upper frequency and lower frequency are taken as input parameters for calculation. Formulas for calculation is based on aforementioned equations. Algorithm used while calculating in Matlab is as follows:

1. Start

2. Enter values of $\tau, \sigma$, and lower and upper cut-off frequency

3. Calculate bandwidth $(\mathrm{B})=$ upper frequency/ lower frequency

4. Calculate active region bandwidth $\left(\mathrm{B}_{\mathrm{ar}}\right)$

5. Calculate maximum wavelength $=$ speed of EM wave in vacuum / lower frequency

6. Calculate number of elements $(\mathrm{N})$

7. Calculate characteristics impedance $\left(\mathrm{Z}_{0}\right)$

8. Calculate separation of two elements.

9. Calculate length of elements: $\mathrm{L}_{\mathrm{i}}=\tau \mathrm{L}_{\mathrm{i}+1}$

10. Calculate spacing between the elements: $R i-R i+1=(L i-L i+1) * \frac{4 \sigma}{1-\tau}$.

11. Repeat step 9 and 10 for 16 times.

12. Calculate SWR

13. Display all results.

14. End

Calculations of parameters using program yields output variables as follow:

Upper cutoff frequency $\left(\mathrm{f}_{2}\right)=150 \mathrm{MHz}$

Lower cutoff frequency $\left(\mathrm{f}_{1}\right)=30 \mathrm{MHz}$

Bandwidth $(\mathrm{B})=\frac{f_{2}}{f_{1}}=5$

Antenna impedance for maximum power transfer and directivity $\left(R_{0}\right)=50 \mathrm{ohm}$

Gain $(\mathrm{G})=8 \mathrm{dBi}$

Relative spacing $(\sigma)=0.175$

Design constant or Periodicity $(\boldsymbol{\tau})=0.865$

Longest length of dipole $\left(\mathrm{L}_{1}\right)=4.9965 \mathrm{~m}$.

Active region bandwidth $\left(\mathrm{B}_{\mathrm{ar}}\right)=1.82765$

Structure bandwidth $\left(\mathrm{B}_{\mathrm{s}}\right)=9.13825$

Number of dipole elements $(\mathrm{N})=16$

Separation between booms $=6.887 \mathrm{~cm}$

$\mathrm{Z}_{\mathrm{avg}}=363.686$

Feeder characteristic Impedance $\left(Z_{0}\right)=238.3072$

As the number of elements was found to be 16 for our frequency range, sixteen dimensions of elements and spacing between them is calculated and tabulated as below: 
Table 1: Length of half element of antenna element varying logarithmically. Length of element is taken half as antenna contains two booms.

\begin{tabular}{|cccc|}
\hline $\begin{array}{c}\text { Element } \\
\left(\mathbf{L}_{\mathbf{i}}\right)\end{array}$ & $\begin{array}{c}\text { Length of half } \\
\text { element(cm) }\end{array}$ & $\begin{array}{c}\text { Element } \\
\left(\mathbf{L}_{\mathbf{i}}\right)\end{array}$ & $\begin{array}{c}\text { Length of half } \\
\text { element(cm) }\end{array}$ \\
\hline $\mathrm{L}_{1}$ & 249.825 & $\mathrm{~L}_{9}$ & 78.3 \\
$\mathrm{~L}_{2}$ & 216.098 & $\mathrm{~L}_{10}$ & 67.729 \\
$\mathrm{~L}_{3}$ & 186.925 & $\mathrm{~L}_{11}$ & 58.586 \\
$\mathrm{~L}_{4}$ & 161.862 & $\mathrm{~L}_{12}$ & 50.677 \\
$\mathrm{~L}_{5}$ & 139.862 & $\mathrm{~L}_{13}$ & 43.835 \\
$\mathrm{~L}_{6}$ & 120.98 & $\mathrm{~L}_{14}$ & 37.981 \\
$\mathrm{~L}_{7}$ & 104.648 & $\mathrm{~L}_{15}$ & 32.799 \\
$\mathrm{~L}_{8}$ & 90.520 & $\mathrm{~L}_{16}$ & 28.371 \\
\hline
\end{tabular}

Table 2: Spacing between elements varying logarithmically

\begin{tabular}{|cccc|}
$\begin{array}{c}\text { Spacing between } \\
\text { elements }\left(\mathbf{R}_{\left.\mathbf{i}-\mathbf{R}_{\mathbf{i}+\mathbf{1}}\right)}\right.\end{array}$ & Spacing(cm) & $\begin{array}{c}\text { Spacing between } \\
\text { elements }\left(\mathbf{R}_{\mathbf{i}}-\mathbf{R}_{\mathbf{i}+\mathbf{1}}\right)\end{array}$ & Spacing (cm) \\
\hline $\mathrm{R} 1-\mathrm{R} 2$ & 87.44 & $\mathrm{R} 9-\mathrm{R} 10$ & 27.41 \\
$\mathrm{R} 2-\mathrm{R} 3$ & 75.63 & $\mathrm{R} 10-\mathrm{R} 11$ & 23.70 \\
$\mathrm{R} 3-\mathrm{R} 4$ & 65.42 & $\mathrm{R} 11-\mathrm{R} 12$ & 20.50 \\
$\mathrm{R} 4-\mathrm{R} 5$ & 56.59 & $\mathrm{R} 12-\mathrm{R} 13$ & 17.74 \\
$\mathrm{R} 5-\mathrm{R} 6$ & 48.95 & $\mathrm{R} 13-\mathrm{R} 14$ & 15.34 \\
$\mathrm{R} 6-\mathrm{R} 7$ & 42.34 & $\mathrm{R} 14-\mathrm{R} 15$ & 13.27 \\
$\mathrm{R} 7-\mathrm{R} 8$ & 36.63 & $\mathrm{R} 15-\mathrm{R} 16$ & 11.48 \\
$\mathrm{R} 8-\mathrm{R} 9$ & 31.68 & & \\
\hline
\end{tabular}

\section{DESIGN IMPLEMENTATION}

Two booms of dimension 1"X1" was used to fabricate elements. Feeder tube and antenna elements both are aluminum tubes. Elements were mounted on the boom using barrel nut of sliding lock and fixed using M-seal. Each half element was mounted on the boom maintaining equal spacing on both the booms in a zigzag manner so that single feeder on the antenna booms provides alternative polarity. Screws and aluminum foils were used for mounting the bolt on the boom and attaching the elements on the bolt.

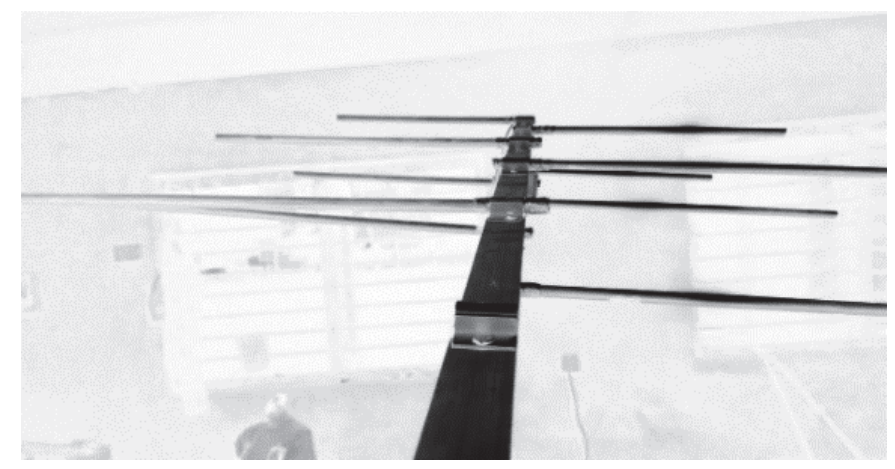

Figure 1: Antenna Fabrication. Barrel bolt was mounted on boom and antenna elements were fixed on the bolt using M-seal and aluminum tape. 
The antenna designed was fed with $75 \mathrm{ohm}$ coaxial cable with a balun. Balun is basically a transformer, used to connect balanced line with unbalanced one. Balun provides impedance transformation in addition to conversion between balanced and unbalanced signal modes; others provide no impedance transformation. For 1:1 baluns (no impedance transformation), the input and output are usually both 50 ohms and $75 \mathrm{ohms}$. The most common impedance-transformation ratio is $1: 4$ (alternatively $4: 1$ ). Some baluns provide other impedance-transformation ratios, such as 1:9 (and 9:1), 1:10 (and 10:1), or 1:16 (and 16:1). Impedance-transformer baluns having a 1:4 ratio are used between systems with impedances of 50 or 75 ohms (unbalanced) and 200 or 300 ohms (balanced).

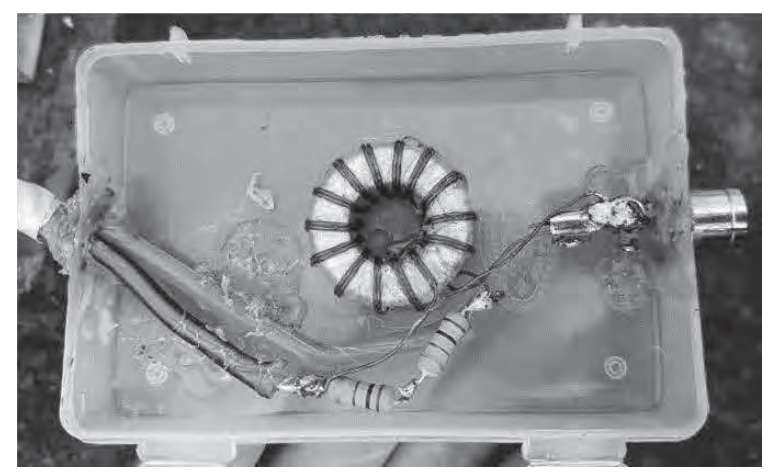

Figure 2: Impedance matching balun constructed with ferrite core and insulated copper wire. [6]

The overall fabricated structure is as shown below:

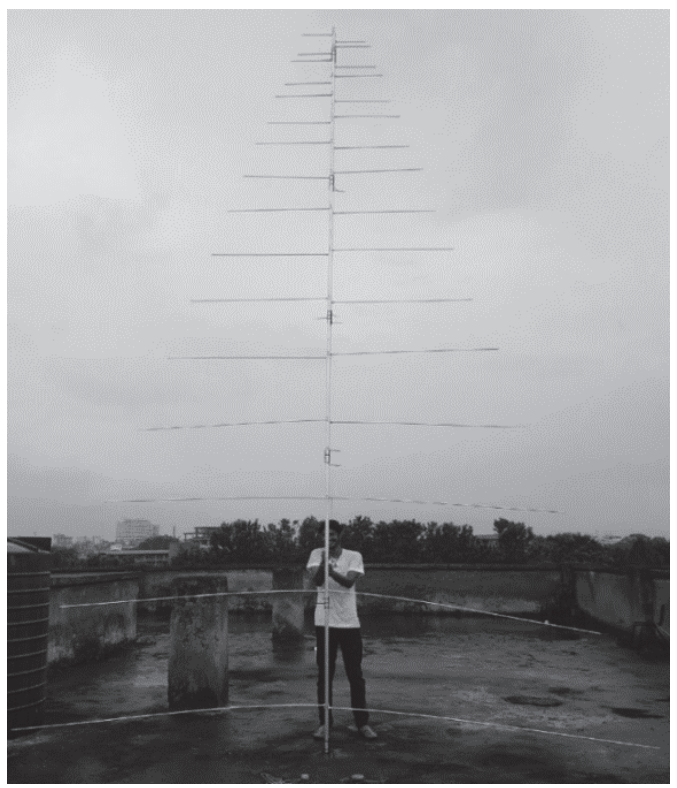

Figure 3: Fully fabricated antenna installed on Department of Electronics and Computer Engineering, Thapathali Campus, Kathmandu. 


\section{DESIGN ANALYSIS}

Vector Network Analyzer (VNA) is an instrument that measures the network parameters of an electrical network such as characteristic impedance in complex form along with its absolute value and Standing Wave Ratio (SWR) at tuned frequency. VNA used in our project was Color Graphic Antenna Analyzer SA-250. It was used to measure the characteristic impedance of LPDA antenna. In addition to this, it was also helpful in determining whether the signal was transmitted from balanced to unbalanced line with low SWR or not. To measure the characteristic impedance of the antenna and confirm the proper operation of the balun, the VNA was connected to the feed point using SMA connector extending from $50 \mathrm{Ohm}$ coaxial cable. The output of balun was connected to the VNA using SMA connector extending from $75 \mathrm{Ohm}$ coaxial cable. The average SWR was measured at 16 different frequencies with an average of 1.623 while the impedance of the antenna was averaged to $71.5625 \mathrm{ohm}$.

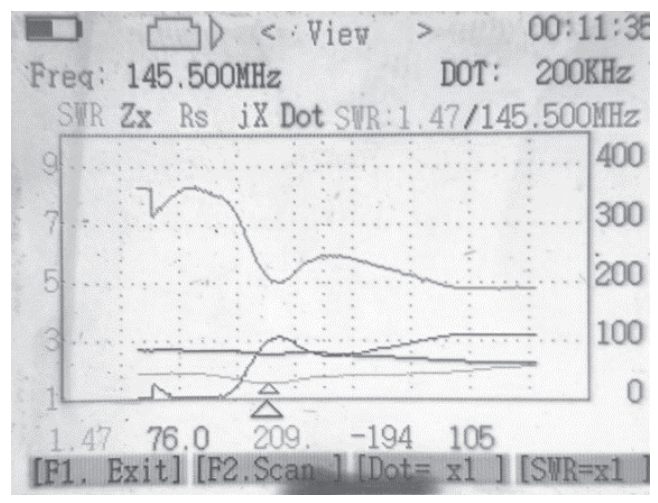

Figure 4: Antenna Analyzer showing properties of antenna at frequency of $145.5 \mathrm{MHz}$.

\section{CONCLUSION}

The designed Log Periodic Dipole Array Antenna can be used as receiving or transmitting antenna for high bandwidth signal transmission. It has frequency range of $30 \mathrm{MHz}$ to $150 \mathrm{MHz}$. The value of SWR and impedance was found to be in the acceptable range.

The error on SWR and Impedance were $8.2 \%$ and $4.58 \%$ respectively. The capacitive effect due to separation between the booms introduced the error on Impedance, whereas the error on SWR was as a result of imperfect impedance matching.

The working range of antenna is in Very High Frequency (VHF) band. In VHF band, LPDA is suitable as a common receiving antenna for different kind of signals like radio signals of range $80 \mathrm{MHz}$ to 108 $\mathrm{MHz}$, Navigation Aids and Aviation controls working respectively on frequency range of $108 \mathrm{MHz}$ to $118 \mathrm{MHz}$ and $118 \mathrm{MHz}$ to $135 \mathrm{MHz}$. Furthermore, the antenna can be used as receiving antenna for solar radio outbursts that occur at the working frequency range of the antenna. For the detection of solar radio outbursts the antenna was placed on open space facing the sun. The receiver system connected to it was used to monitor the change in intensity pattern of the radio outbursts then predict solar flares and radio blackouts. 


\section{ACKNOWLEDGEMENT}

We would like to thank our project supervisor Er. Saroj Shakya and Mr. Sunil Sera Karki for all the guidance they had provided. We would also like to acknowledge with much appreciation the help provided by Orion Space Nepal. Finally, we are very thankful to the Department of Electronics and Computer Engineering, IOE Thapathali Campus for providing us workspace.

\section{REFERENCES}

[1] C. A. Balanis, Antenna Theory Analysis and Design, Hobokin, New Jersey: A John Wiley and sons.

[2] D. Isbell, R. DuHamel, Broadband logarithmically periodic antenna structures, New York: IEEE, 1966.

[3] R. L. Carrel, "Analysis and Design of Log Periodic Dipole Antenna," Electrical Engineering Department, University of Illioins, Ann Arbor, 1961.

[4] J. B. V. B. Frantisek Hutira, "Design and Investigation of a log-periodic antenna for DCS, PCS and UMTS mobile communications bands".

[5] V. S. Kale, "Impedance and Power Loss Characteristics of," International Journal of Advanced Research in Computer and Communication Engineering, vol. 5, no. 4, pp. 625-628, 2016.

[6] J. Sevick, Transmission Line Transormers Handbook, Amidon Associates Inc.. 